\title{
Subjektives Wohlbefinden und Arbeitsfähigkeit von Krankenhauspersonal nach SARS-CoV-2-Immunisierung mit dem mRNS-Impfstoff BNT162b2 - Ergebnisse einer anonymen Umfrage Subjective well-being and ability to work of hospital staff after SARS-CoV2 immunization with the mRNA vaccine BNT162b2
}

(ㄷ) (1) $(2) \Theta$

Autoren

Johannes Kalbhenn ${ }^{1}$, Thorsten Hammer ${ }^{2}$, Martin Hug $^{3}$, Daniel Dürschmied ${ }^{4}$, Frederik Wenz ${ }^{5}$, Daniel Steinmann ${ }^{6}$

Institute

1 Klinik für Anästhesiologie und Intensivmedizin, Universitätsklinikum Freiburg

2 Ärztlicher Leiter Chirurgie Notfallzentrum und Zentrales Impfzentrum, Universitätsklinikum Freiburg

3 Leitender Klinik-Apotheker, Universitätsklinikum Freiburg

4 Department Innere Medizin, Medizinische Intensivtherapie, Universitätsklinikum Freiburg

5 Leitender Ärztlicher Direktor, Universitätsklinikum Freiburg

6 Leitender Betriebsarzt, Universitätsklinikum Freiburg

Schlüsselwörter

COVID-19, Impfung, SARS-CoV-2, Nebenwirkung,

Arbeitsunfähigkeit, Personalplanung

Key words

COVID-19, vaccination, SARS-CoV2, side effects, incapacity for work, personnel planning

online publiziert 09.07 .2021

Bibliografie

Dtsch Med Wochenschr 2021; 146: e58-e64

DOI 10.1055/a-1520-2127

ISSN 0012-0472

(C) 2021. The Author(s).

This is an open access article published by Thieme under the terms of the Creative Commons Attribution-NonDerivative-NonCommercial License, permitting copying and reproduction so long as the original work is given appropriate credit. Contents may not be used for commecial purposes, or adapted, remixed, transformed or built upon. (https://creativecommons.org/licenses/by-nc-nd/4.0/)

Georg Thieme Verlag KG, Rüdigerstraße 14,

70469 Stuttgart, Germany

Korrespondenzadresse

PD Dr. med. Johannes Kalbhenn

Klinik für Anästhesiologie und Intensivmedizin

Universitätsklinikum Freiburg, Hugstetter Straße 55,

79106 Freiburg im Breisgau, Deutschland

Tel.: $+49 / 761 / 27023121$

Fax: $+49 / 761 / 27023400$

Johannes.kalbhenn@uniklinik-freiburg.de

\section{ZUSAMMENFASSUNG}

Einleitung Seit dem 27.12.2020 wurden in Deutschland unter anderem Mitarbeiterlnnen des Gesundheitssystems mit dem Vakzin BNT162b2 gegen das SARS-Coronavirus-2 geimpft. Erste Beobachtungen zeigten, dass es insbesondere bei jüngeren Geimpften zu Nebenwirkungen kommen kann. In dieser Studie wurde am Beispiel von Klinikmitarbeiterlnnen das selbstempfundene Wohlbefinden nach der jeweils ersten und zweiten Dosis des Impfstoffes untersucht.

Methoden Anonymisierter Online-Fragebogen, einmalig ausfüllbar durch alle MitarbeiterInnen nach Erhalt der zweiten Dosis von BNT162b2. Die Ausprägung von Nebenwirkungen wurde über eine ordinale numerische Rating-Skala mit Werten zwischen 0 und 10 abgefragt. Weitere wesentliche Datenpunkte waren Alter, Geschlecht und Berufsgruppe. Die Arbeitsfähigkeit in den Folgetagen der Injektionen wurde ebenfalls durch Selbstangabe erhoben.

Ergebnisse Es wurden Daten von 555 Befragten ausgewertet. Das mittlere Alter lag bei 40,25 Jahren (Standardabweichung 12,35). $56 \%$ der Befragten waren weiblich, $44,3 \%$ gehörten zum ärztlichen, 42,9\% zum pflegerischen Dienst und 12,8\% waren anderen Berufsgruppen mit COVID-19-Patientenkontakt zuzuordnen. Etwa $2 \%$ aller Mitarbeiter verspürten überhaupt keine Nebenwirkungen. Häufigste Nebenwirkung waren Schmerzen an der Injektionsstelle. In absteigender Häufigkeit folgten Müdigkeit, Kopfschmerzen und Myalgien. Nach der ersten Dosis gaben 3 Viertel der Befragten an, die Impfung insgesamt gut vertragen zu haben, nach der zweiten Dosis war es nur noch die Hälfte. Nach der ersten Dosis fühlten sich über $90 \%$ der Befragten am Folgetag wieder arbeitsfähig, nach der zweiten Dosis gab ein Drittel an, erst am zweiten Tag wieder arbeitsfähig gewesen zu sein. 2,2\% aller MitarbeiterInnen mussten sich nach der ersten, 19,5\% nach der zweiten Dosis für mindestens einen Tag arbeitsunfähig melden.

Schlussfolgerung Die Impfung mit BNT162b2 führte - insbesondere nach der zweiten Dosis - häufig zu Nebenwirkungen. Diese hatten in 19\% der Befragten eine Krankmeldung 
nach der zweiten Dosis zur Folge. Dennoch würden sich $95 \%$ aller Befragten wieder für eine Coronavirus-Impfung entscheiden.

\section{ABSTRACT}

Background Since December 27, 2020, employees of the health system in Germany have been vaccinated against the SARS coronavirus- 2 with the vaccine BNT162B2. Initial observations show that especially among younger vaccinated people side effects are common. In this study, using the example of clinic employees, the self-perceived well-being after the first and second dose of the vaccine was examined.

Methods Anonymized online questionnaire to be filled out once by all employees after the second dose of BNT162B2 was offered. The severity of side effects was queried using an ordinal numerical rating scale with values between 0 and 10 . Other key data points were age, gender, and occupational group. The ability to work in the days following the injections was recorded by self-reporting.

Results Data from 555 respondents were evaluated. The mean age was 40.25 years (standard deviation 12.35 ). $56 \%$ of the respondents were female, $44.3 \%$ belonged to the medical service, $42.9 \%$ to the nursing service and $12.8 \%$ were assigned to other professional groups with COVID-19 patient contact. Around $2 \%$ of all employees did not experience any side effects at all. The most common side effect was pain at the injection site. Fatigue, headaches and myalgia followed with decreasing frequency. After the first dose, $3 / 4$ of the respondents said they had tolerated the vaccination well overall, after the second dose it was only half. After the first dose, over $90 \%$ of the respondents felt that they were able to work again on the following day, after the second dose one third stated that they were only able to work again on the second day. $2.2 \%$ of all employees had to report that they were unable to work for at least one day after the first dose and $19.5 \%$ after the second dose.

Conclusions Vaccination with BNT162B2 frequently leads to side effects, especially after the second dose. Perception of side effects resulted in $19 \%$ of those questioned being sick after the second dose. Nevertheless, $95 \%$ of all respondents would choose a coronavirus vaccination again.

\section{Einleitung}

Im Rahmen der weltweiten SARS-CoV-2-Pandemie wurden erstmalig Ribonukleinsäure (RNS) -basierte Vakzine bis zur Massenanwendung entwickelt. Der für das Spike-Protein des SARS-CoV-2Virus kodierende Impfstoff BNT162b2 wurde durch den Austausch einzelner Nukleotide durch natürlich vorkommende Isomere modifiziert [1, 2]. Diese mRNS wird in Lipid-basierte Nanopartikel (LPN) „verpackt“ [3], um die Penetration durch die Zellmembran zu erleichtern, und durch Zusatz von PolyethylenGlykol (PEG) -Lipidkonjugat zusätzlich stabilisiert [4]. Durch die Zielzellen - zum Beispiel Muskelzellen im Bereich der Injektionsstelle - wird das für sich allein ungefährliche Spike-Protein des Virus synthetisiert und löst in der Folge die spezifische Immunreaktion mit Bildung von Antikörpern aus.

In einer Phase-III-Studie mit mehr als 37000 Probanden wurde eine Wirksamkeit von $95 \%$ festgestellt. Dafür sind 2 intramuskuläre Injektionen von je $30 \mu \mathrm{g}$ Wirkstoff im Abstand von 3 Wochen notwendig. Jedoch wurden neben der guten Wirksamkeit auch häufige Nebenwirkungen berichtet: 70-80\% aller Probanden klagten über milden oder moderaten Schmerz an der Einstichstelle, zwischen 30 und $40 \%$ über Müdigkeit und Kopfschmerzen. 14-37\% beklagten Gliederschmerzen. Die Nebenwirkungen waren jeweils nach der zweiten Injektion häufiger, die Altersgruppe unter 55 Jahren war stärker betroffen [4].

Am 21.12.2020 erhielt das Vakzin die Zulassung durch die Europäische Arzneimittelbehörde (EMA). Nach Chargenfreigabe durch das Paul-Ehrlich-Institut war am 27.12.2020 der offizielle Impfstart in Deutschland. Gemäß der „Verordnung zum Anspruch auf Schutzimpfung gegen das Coronavirus SARS-CoV-2“ wurden Personen „höchster Priorität“ immunisiert. Das Impfzentrum Freiburg nahm direkt am 27.12.2020 seine Arbeit auf. Parallel zu
Menschen über 80 Jahren wurden auch Mitarbeiterlnnen des Gesundheitssystems geimpft, ein Teil davon aus dem Universitätsklinikum Freiburg. Diese Studie wurde initiiert, um selbstberichtete Nebenwirkungen sowie das subjektive Befinden und die selbst eingeschätzte Arbeitsfähigkeit in den ersten Tagen nach den jeweiligen Injektionen zu erfassen. Es wurde angenommen, dass die zweite Dosis insgesamt schlechter vertragen wird als die erste. Außerdem sollten Einschätzungen über die Häufigkeit von Krankheitsgefühl getroffen sowie die Häufigkeit tatsächlich erfolgter Arbeitsunfähigkeitsmeldung bestimmt werden.

\section{Methode}

Die ersten MitarbeiterInnen der Corona-Stationen und der Notaufnahme des Universitätsklinikums wurden am 27.12.2020 mit der ersten Dosis immunisiert, ab dann wurden täglich weitere Termine an Mitarbeiterlnnen der höchsten Prioritätsstufe vergeben. Die zweite Dosis wurde jeweils exakt 21 Tage später verabreicht. Als Erfassungsinstrument für diese Querschnittsstudie wurde von der Ethikkommission der Albert-Ludwigs-Universität Freiburg am 26.01.2021 eine anonyme Umfrage vorgeschlagen und als unbedenklich bewertet. Der Personalrat stimmte dieser Erfassung am 28.01.2021 ebenfalls zu.

Die Erstellung des Fragebogens erfolgte unter Beratung durch eine Psychologin. Die Ausprägung von Nebenwirkungen wurde über eine ordinale numerische Rating-Skala mit Werten zwischen 0 (trifft nicht zu) und 10 (trifft voll zu) abgefragt [5]. Weitere wesentliche Datenpunkte waren Alter, Geschlecht und Berufsgruppe. Die Arbeitsfähigkeit in den Folgetagen der Injektionen wurde ebenfalls durch Selbstangabe erhoben. Anschließend wurde der Fragebogen als „Online“-Modul für das Browser-basierte Intranet und als Modul für die klinikeigene „MeineUniklinik“-App formatiert. In einem Pre- 
test mit 10 Personen wurde überprüft, ob alle Antwortmöglichkeiten berücksichtigt wurden und ob die Fragen und Antwortmöglichkeiten einfach und verständlich waren. Es wurde anschließend beurteilt, ob in Zahlenwerten wirklich das abgebildet wurde, was die Pretest-Teilnehmer auch aussagen wollten. Die Datensätze des Pretests wurden nicht in die Gesamtanalyse inkludiert. Durch eine Fangfrage wurden unplausible Datensätze identifiziert und von der Analyse ausgeschlossen. Zur Validierung wurde der finale Gesamtdatensatz zufällig geteilt und die zentralen Tendenzen der Angaben/Einschätzungen miteinander verglichen. Dabei ergaben sich keine Unterschiede.

Vom 29.01.2021-11.02.2021 wurde der Online-Fragebogen allen MitarbeiterInnen zur Verfügung gestellt. Dieser konnte mit den persönlichen Login-Daten jeweils nur einmal ausgefüllt werden. Es wurde dazu aufgerufen, erst nach Abklingen eventueller Nebenwirkungen nach der zweiten Dosis teilzunehmen. Die unberechtigte Teilnahme wurde durch eine Plausibilitätsfragestellung erschwert. Die Datensätze wurden ohne Verbindung mit den Login-Daten in einer Datenbank gespeichert und anschließend als „Excel“-Tabelle (Microsoft, Redmond, Washington, USA) ausgegeben und weiter prozessiert.

Die statistische Analyse erfolgte mit GraphPad Prism (GraphPad Software, San Diego, CA). Für Vergleiche innerhalb der ersten Impfung wurden für nichtparametrische ordinale Daten der KruskalWallis-Test und - wenn indiziert - der Mann-Whitney-Test verwendet. Für den Vergleich der Unterschiede zwischen erster und zweiter Impfdosis wurden für die nichtparametrischen Daten der Wilcoxon-Test für ordinale und der McNemar-Test für binäre Skalen angewendet. Als Signifikanzwert für Unterschiede zwischen erster und zweiter Impfung wurde ein p-Wert von < 0,01 angenommen. Zur Vermeidung einer Alphafehler-Kumulierung bei multiplen Vergleichen wurde eine Bonferroni-Korrektur durchgeführt.

\section{Ergebnisse}

573 Mitarbeiterlnnen haben den Fragebogen innerhalb des Observationszeitraums von 2 Wochen online beantwortet. 18 Datensätze wurden wegen nicht plausibler oder unvollständiger Angaben ausgeschlossen, die finale Stichprobe bestand aus 555 Befragten. Das durchschnittliche Alter lag bei 40,25 Jahren (Standardabweichung 12,35). $56 \%$ der Befragten waren weiblich, $44,3 \%$ gehörten zum ärztlichen, 42,9\% zum pflegerischen Dienst und 12,8\% waren anderen Berufsgruppen mit COVID-19-Patientenkontakt zuzuordnen. Etwa $2 \%$ aller Geimpften berichteten weder bei der ersten noch bei der zweiten Dosis von Nebenwirkungen (5,6\% hatten keine Nebenwirkungen bei der ersten und 3,6\% bei der zweiten Impfung). Als häufigste Nebenwirkung wurden Schmerzen an der Injektionsstelle bemerkt, mit etwas über $89 \%$ war die Inzidenz nach erster und zweiter Dosis nicht unterschiedlich $(p=0,749)$. In absteigender Häufigkeit wurden Müdigkeit, Kopfschmerzen und Myalgien angegeben ( $\triangleright$ Abb. 1). Diese waren nach der zweiten Impfdosis jeweils signifikant häufiger und intensiver als nach der ersten (jeweils $p<0,001$ ). Nach der ersten Dosis gaben 3 Viertel der Befragten an, die Impfung insgesamt gut vertragen zu haben, nach der zweiten Dosis war es nur noch die Hälfte ( $\bullet$ Abb. 2). Schmerzen an der Injektionsstelle wurden sowohl nach der ersten als auch nach der zweiten Dosis signifikant häufiger von den bis zu
39-Jährigen im Vergleich zu den 50-69-Jährigen angegeben. Dies traf auch für die Müdigkeit nach der zweiten Dosis zu. Unterschiede zwischen den Geschlechtern oder den Berufsgruppen bestanden diesbezüglich nicht $(\triangleright$ Tab. 1, 2).

Nach der ersten Dosis fühlten sich über $90 \%$ der Befragten am Folgetag wieder arbeitsfähig, nach der zweiten Dosis gab ein Drittel an, erst am zweiten Tag wieder arbeitsfähig gewesen zu sein, über $10 \%$ benötigten mehr als 2 Tage zur Rekonvaleszenz ( $\triangleright$ Abb. 3). 2,2\% aller MitarbeiterInnen mussten sich nach der ersten, 19,5\% nach der zweiten Dosis für mindestens einen Tag arbeitsunfähig melden $(p<0,001)(\triangleright$ Abb. 4).

Geschlechterspezifische oder altersabhängige Unterschiede bestanden bezüglich der Arbeitsunfähigkeit nicht. Es konnten keine Unterschiede zwischen den einzelnen Berufsgruppen ausgemacht werden. Keine lebensbedrohliche Nebenwirkung (Anaphylaxie o. ä.) wurde im Zusammenhang mit der Impfung beobachtet oder später an den betriebsärztlichen Dienst gemeldet.

\section{Diskussion}

In dieser Studie wurden Nebenwirkungen und Arbeitskraftausfälle in einer deutschen Universitätsklinik durch anonyme Befragung von Geimpften erhoben. Selbstberichtete Nebenwirkungen nach der Immunisierung mit BNT162b2 waren mit $21 \%$ nach der ersten und über $50 \%$ bei der zweiten Dosis häufig. Dabei spielten vor allem lokale Nebenwirkungen wie Schmerzen an der Injektionsstelle eine große Rolle. Systemische Wirkungen wie Müdigkeit, Kopfschmerzen, Arthralgien und Myalgien sind am ehesten die Folge der Immunantwort und waren häufiger ausgeprägt als in der Phase-III-Zulassungsstudie [4], was sich mit dem geringeren Durchschnittsalter in unserer Stichprobe erklären lässt. Neben den abgefragten Nebenwirkungen wurden im Freitext Lymphknotenschwellungen, Schlafstörungen und Übelkeit angegeben. All diese Nebenwirkungen sind auch für andere Impfungen, wie z. B. die Immunisierung gegen Influenza, beschrieben.

Insgesamt wurde in der Selbsteinschätzung der Mitarbeiterlnnen die zweite Dosis signifikant schlechter vertragen als die erste, was sich bei gleicher Wirkstoffmenge durch die gesteigerte Immunreaktion erklären lässt. Eine niedrige Krankmelderate nach der ersten Impfung sollte nicht darüber hinwegtäuschen, dass sich nach der zweiten Dosis fast $40 \%$ der Befragten erst am zweiten und dritten Tag wieder arbeitsfähig gefühlt und sich mehr als 19\% der Mitarbeiter krankgemeldet hatten. Zwei Drittel der Krankmeldungen betrafen nur den Tag nach der zweiten Dosis, ein Drittel jedoch betraf bis zu 3, Einzelfälle sogar bis zu 10 Tage nach Injektion. Dies sollte bei der Planung von Impfkampagnen in sensiblen Bereichen beachtet werden, ein gestaffeltes Vorgehen mit mindestens 3 Tagen Abstand scheint hier sinnvoll zu sein.

Bis zum 02.04.2021 wurden in Deutschland 10722876 Impfungen mit BNT162b2 durchgeführt. In diesem Zusammenhang wurden 12409 Verdachtsfälle auf unerwünschte Nebenwirkungen an das Robert-Koch-Institut gemeldet, davon 1832 Fälle mit schwerwiegenden Reaktionen. Als schwerwiegende Reaktionen gelten solche, bei denen die Personen im Krankenhaus behandelt werden, oder Reaktionen, die als medizinisch bedeutsam eingeordnet wurden [6]. Die Inzidenz von Nebenwirkungen war in der 


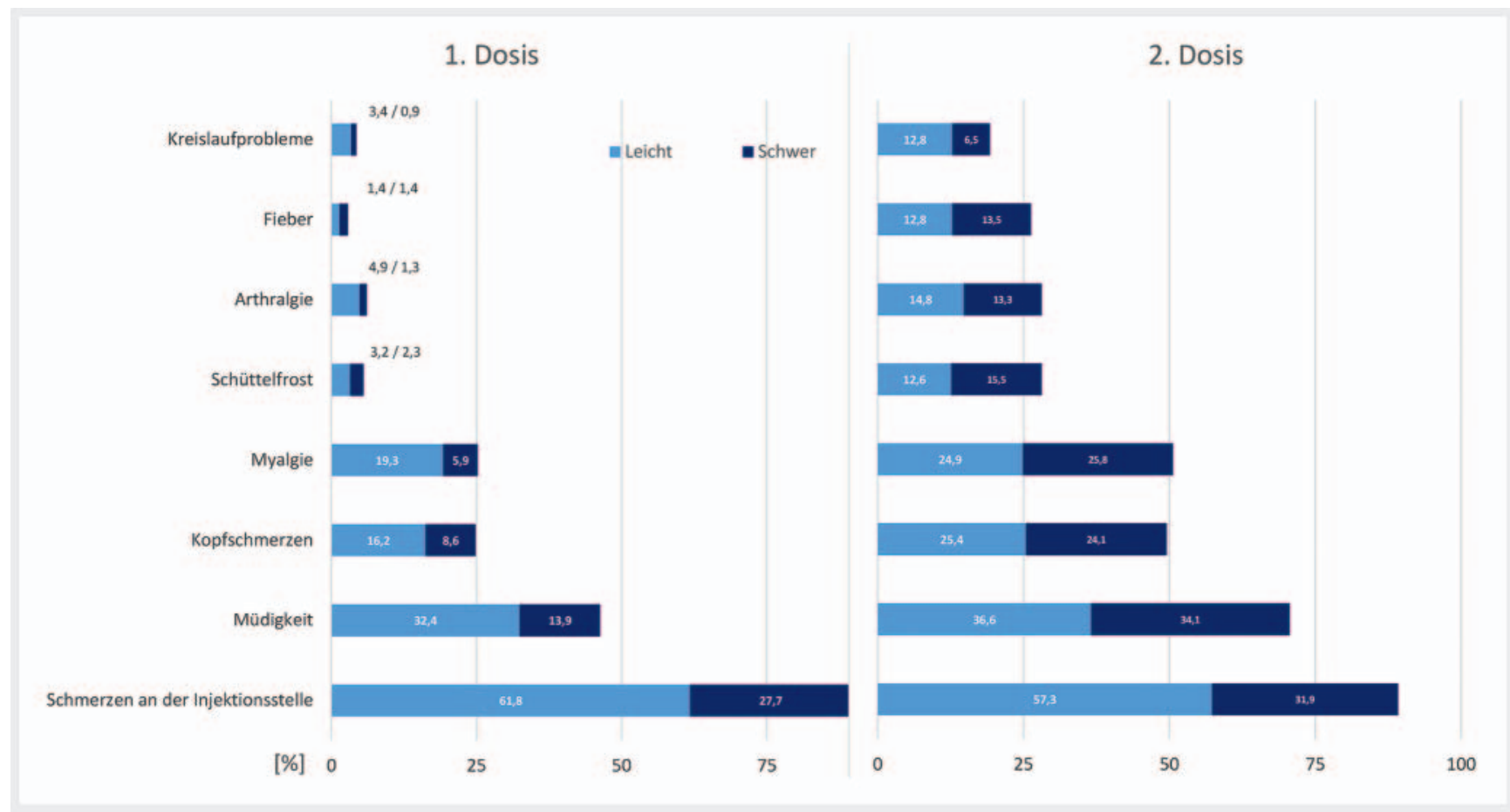

- Abb. 1 Häufigkeit der Nebenwirkungen in \% auf einer Skala von 0-10 (leicht 0-5, hellblau; schwer 6-10 dunkelblau).

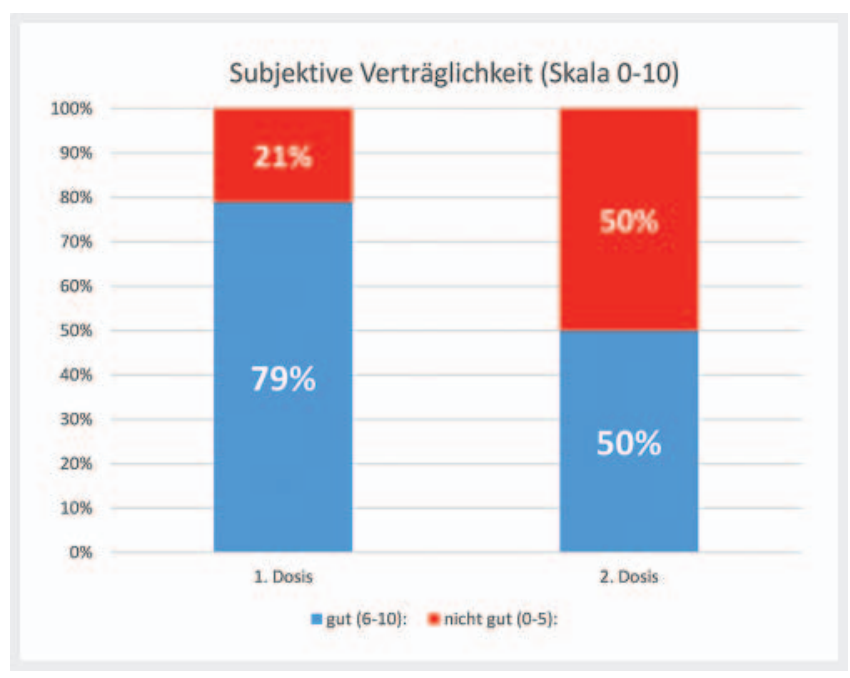

Abb. 2 Subjektive Verträglichkeit der ersten respektive zweiten Dosis auf einer Skala von 0-10 (nicht gut vertragen 0-5, rot; gut vertragen 6-10, blau).

von uns untersuchten Stichprobe höher. Dies liegt unserer Meinung nach an der systematischen Abfrage. Das Register des Robert-Koch-Instituts muss proaktiv aufgerufen werden, um dort Nebenwirkungen zu melden. In der von uns untersuchten Stichprobe trat keine Anaphylaxie auf. Diese ist mit einer Häufigkeit von 4,7 pro 1 Million Impfungen mit BNT162b2 ohnehin sehr selten [7-9].

\section{Limitationen}

Eine einheitliche Dokumentation über die gesamte Population aller geimpfter MitarbeiterInnen wurde wegen Bedenken der zuständigen Ethikkommission und der Personalvertretung nicht angefordert. Insbesondere sollte verhindert werden, dass der Arbeitgeber über eine „Impfliste“ verfügt, um das Vertrauen in die Möglichkeit zur freien Entscheidung pro oder wider Impfung bei den MitarbeiterInnen zu erhalten. Somit kann nicht mit absoluter Sicherheit berechnet werden, ob die mit 555 Befragten große Stichprobe völlig repräsentativ für die gesamte Population ist. Unter Umständen haben MitarbeiterInnen, die keine Nebenwirkungen verspürt haben, weniger Bereitschaft gezeigt, an der Umfrage teilzunehmen („Reporting Bias“). Dem wurde mit mehrfachem Aufruf der Bereichsleitungen und durch Appelle in den jeweiligen Teambesprechungen begegnet. Die zweite Dosis der Impfung lag zum Zeitpunkt der Umfrage weniger lang zurück als die erste, sodass es durch bessere Erinnerbarkeit auch einen „Recall Bias“ geben könnte. Darüber hinaus könnte durch die Befragung von medizinischem Fachpersonal bei den Teilnehmern selbst ein gewisser „Observer Bias“ hinsichtlich möglicher Häufung von Nebenwirkungen bestehen, insbesondere bei der zweiten Dosis. Beide Effekte (Recall und Observer Bias) sollten unserer Meinung nach jedoch durch den selektiven/exklusiven Zugang zu beiden Impfterminen, den zeitlich kurzen Abstand von 3 Wochen zwischen beiden Impfungen sowie eine umfassende Aufklärung über die Impfung selbst und die damit zu erwartenden Wirkungen und Nebenwirkungen im Vorfeld der Impftermine abgeschwächt worden sein. Wegen des anonymen Designs wurde keine Wirksamkeitsüberprüfung, z. B. durch Antikörpertests, vorgenom- 
- Tab. 1 Altersabhängiger Unterschied der häufigsten Nebenwirkungen nach der ersten und zweiten Dosis der Impfung.

\begin{tabular}{|c|c|c|c|c|}
\hline & \multicolumn{2}{|l|}{ 1. Dosis } & \multicolumn{2}{|l|}{ 2. Dosis } \\
\hline & $\begin{array}{l}\text { p-Wert } \\
\text { (Kruskal-Willis-Test) }\end{array}$ & $\begin{array}{l}\text { p-Wert } \\
\text { (Mann-Withney-U-Test) } \\
\text { wenn Kruskal-Willis }<0,01\end{array}$ & $\begin{array}{l}\text { p-Wert } \\
\text { (Kruskal-Willis-Test) }\end{array}$ & $\begin{array}{l}\text { p-Wert } \\
\text { (Mann-Withney-U-Test) } \\
\text { wenn Kuskal-Willis < } 0,01\end{array}$ \\
\hline Schmerz Injektionsstelle & $<0,001$ & $\begin{array}{l}<0,001 \\
\text { für <30-39-Jährige } \\
\text { vs. 50-69-Jährige }\end{array}$ & $<0,001$ & $\begin{array}{l}<0,001 \\
\text { für <30-39-Jährige } \\
\text { vs. 50-69-Jährige }\end{array}$ \\
\hline Müdigkeit & 0,43 & n.a. & $<0,01$ & $\begin{array}{l}<0,01 \\
\text { für <30-Jährige } \\
\text { vs. } 60-69 \text {-Jährige } \\
\text { und } 30-39 \text {-Jährige } \\
\text { vs. } 50-69 \text {-Jährige }\end{array}$ \\
\hline Kopfschmerzen & 0,26 & n.a. & 0,26 & n.a. \\
\hline Muskelschmerz & 0,013 & n.a. & 0,013 & n.a. \\
\hline
\end{tabular}

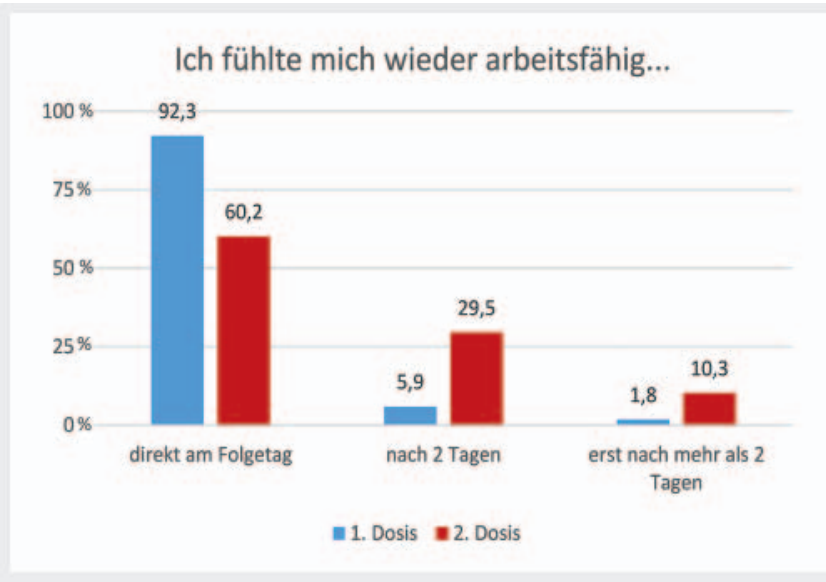

- Abb. 3 Häufigkeit der Selbsteinschätzung zur Arbeitsfähigkeit nach der Impfung in \% (1. Dosis blau; 2 . Dosis rot).

men. Ein positiver Befund hätte die Akzeptanz der Impfung womöglich noch gesteigert.

\section{Schlussfolgerung}

Mit Impfstoffen wie BNT162b2 stehen potente Mittel zur Eindämmung der SARS-CoV-2-Pandemie zur Verfügung. Eine hohe Impfrate ist zeitnah anzustreben. Bei der Planung von Impfkampagnen bei Berufstätigen sollte beachtet werden, dass die Hälfte des geimpften Personals in der Selbstangabe die zweite Dosis des mRNA-Vakzins eher nicht gut vertragen hat und fast $20 \%$ der Arbeitskräfte am Folgetag ausgefallen sind. Erfreulich ist jedoch,

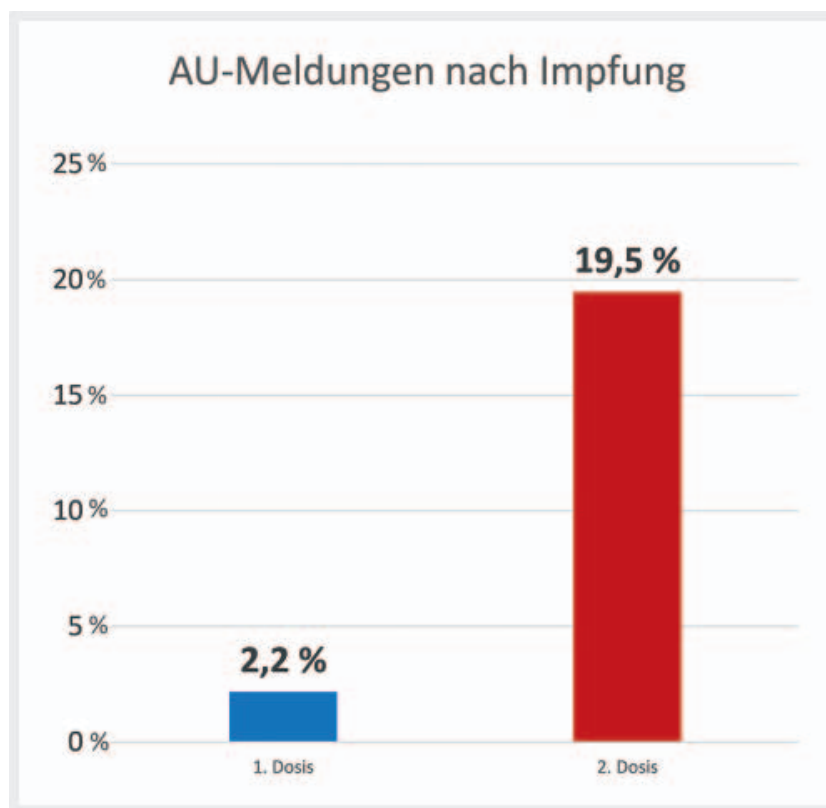

- Abb. 4 Anteil der Befragten mit Arbeitsunfähigkeitsmeldung (AU) nach der ersten und zweiten Dosis in \% (1. Dosis blau; 2. Dosis rot).

dass sich mehr als $95 \%$ der Befragten nach den erlebten Erfahrungen mit der Impfung und vor dem Hintergrund ihrer Expertise als Behandler von COVID-19-Patienten erneut für eine Impfung entscheiden würden ( $\triangleright$ Abb.5). Dies zeigt, dass in der Einschätzung der Geimpften die Vorteile der Impfung den vorübergehenden Dyskomfort durch die Nebenwirkungen überwiegen. 


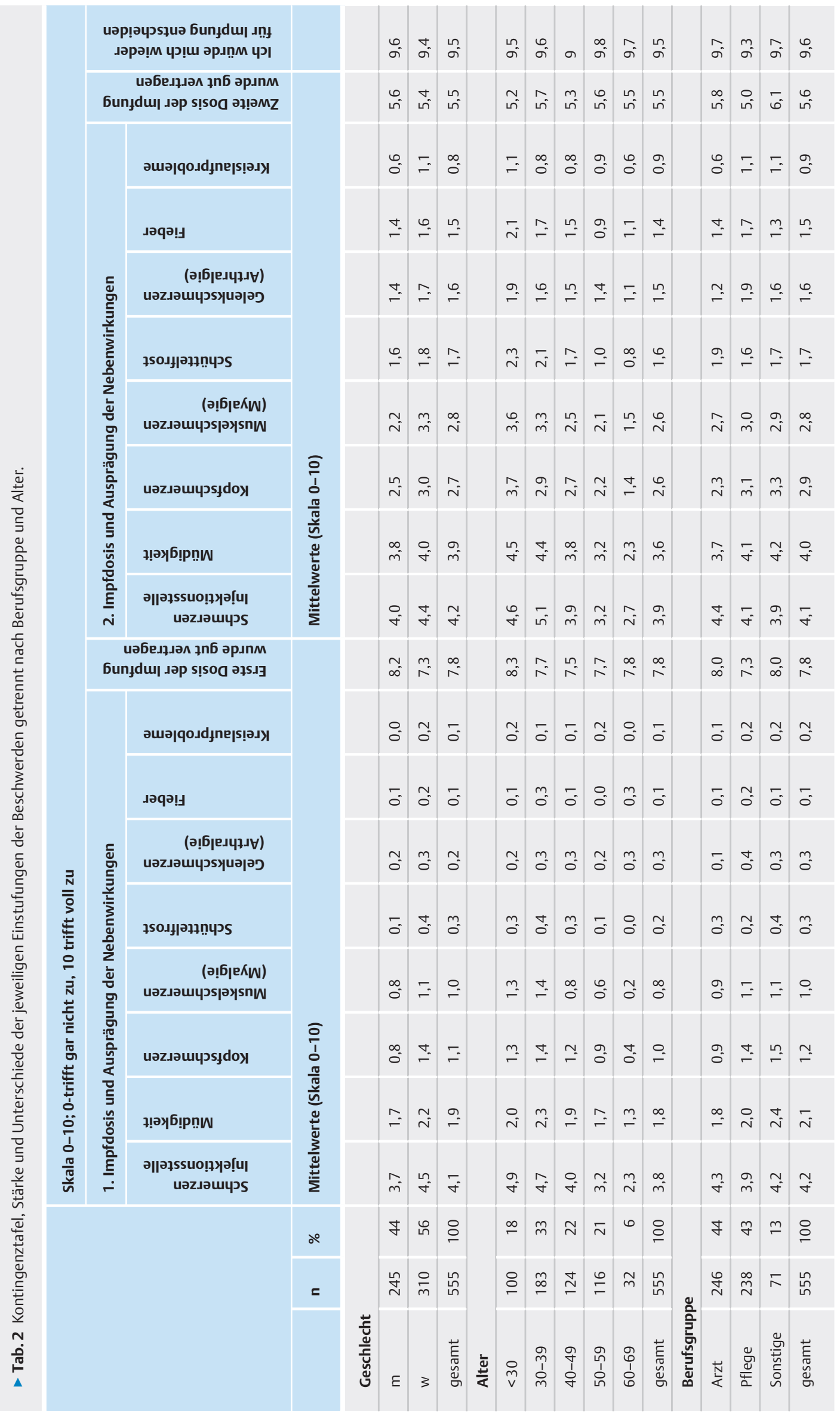




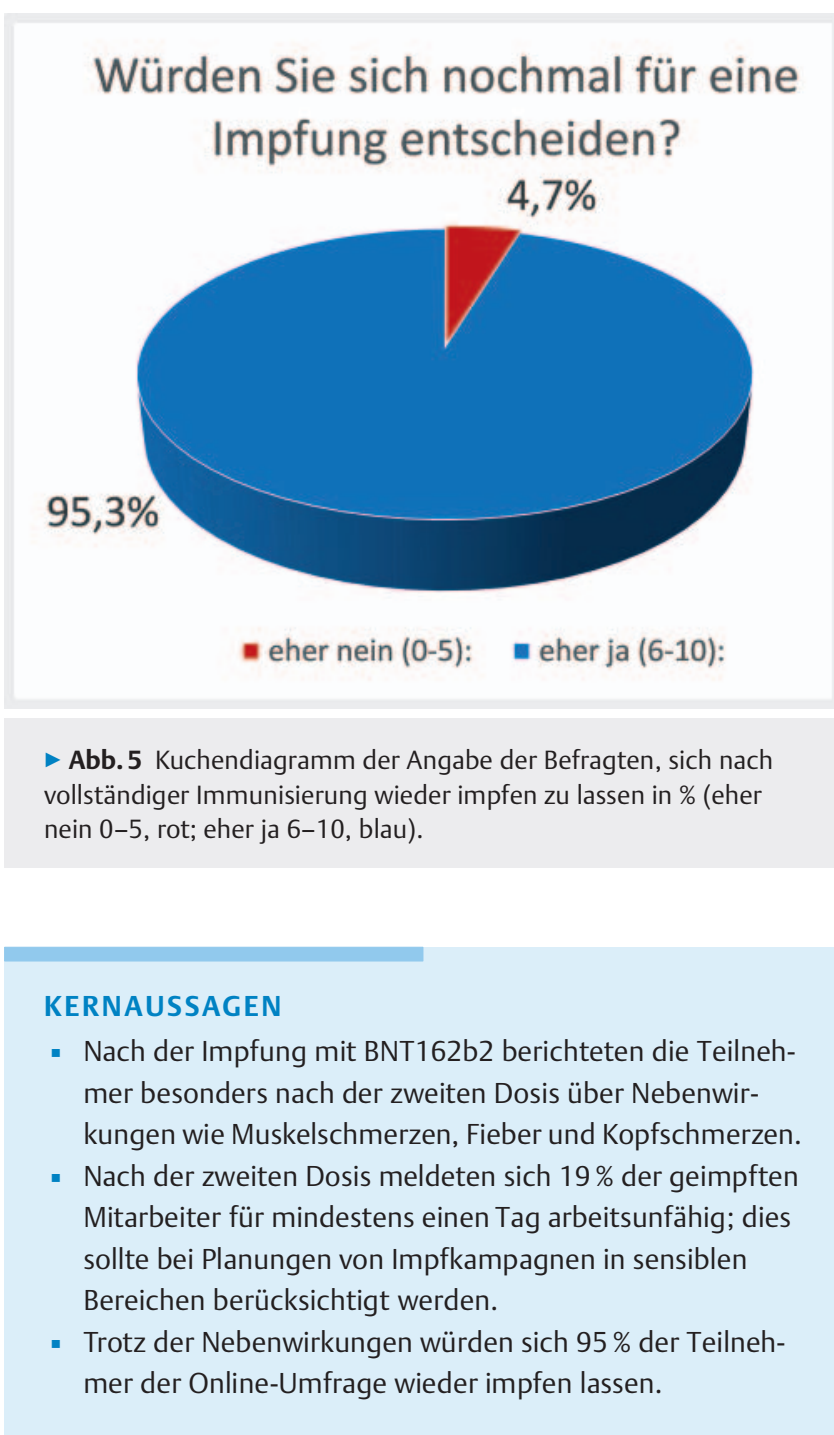

Interessenkonflikt

Die Autoren erklären, dass kein Interessenkonflikt besteht. Die Studie wurde am Universitätsklinikum Freiburg im Breisgau durchgeführt. Die Ethikkommission der Albert-Ludwigs-Universität, der Vorstandsvorsitzende des Universitätsklinikums sowie der Personalrat haben der Durchführung der anonymen Befragung der MitarbeiterInnen im Vorfeld zugestimmt.

\section{Literatur}

[1] Pardi N, Hogan M], Porter FW et al. mRNA vaccines - a new era in vaccinology. Nat Rev Drug Discov 2018; 17 (4): 261-279

[2] Kariko K, Muramatsu H, Welsh FA et al. Incorporation of pseudouridine into mRNA yields superior nonimmunogenic vector with increased translational capacity and biological stability. Mol Ther 2008; 16 (11): 18331840

[3] Pardi N, Tuyishime S, Muramatsu $\mathrm{H}$ et al. Expression kinetics of nucleosidemodified mRNA delivered in lipid nanoparticles to mice by various routes. J Control Release 2015; 217: 345-351

[4] Polack FP, Thomas SJ, Kitchin N et al. Safety and Efficacy of the BNT162b2 mRNA Covid-19 Vaccine. The New England journal of medicine 2020; 383 (27): 2603-2615

[5] Duller C. Einführung in die Statistik mit EXCEL und SPSS. Berlin, Heidelberg: Springer Gabler; 2019. XII, 323p

[6] Robert-Koch-Institut. Sicherheitsbericht Verdachtsfälle von Nebenwirkungen und Impfkomplikationen nach Impfung zum Schutz vor COVID-19 2021. https://www.pei.de/SharedDocs/Downloads/DE/newsroom/ dossiers/sicherheitsberichte/sicherheitsbericht-27-12-bis-02-04-21.pdf Stand 4.4.2021

[7] Shimabukuro TT, Cole M, Su JR. Reports of Anaphylaxis After Receipt of mRNA COVID-19 Vaccines in the US-December 14, 2020-January 18, 2021. Jama 2021; 325 (11): 1101-1102

[8] Banerji A, Wickner PG, Saff RS et al. mRNA Vaccines to Prevent COVID-19 Disease and Reported Allergic Reactions: Current Evidence and Suggested Approach. J Allergy Clin Immunol Pract 2021; 9 (4): 1423-1437

[9] El-Shitany NA, Harakeh S, Badr-Eldin SM et al. Minor to Moderate Side Effects of Pfizer-BioNTech COVID-19 Vaccine Among Saudi Residents: A Retrospective Cross-Sectional Study. Int J Gen Med 2021; 14: 1389-1401 